\title{
Erratum to: Visualization and analysis of EPS glycoconjugates of the thermoacidophilic archaeon Sulfolobus metallicus
}

\author{
Ruiyong Zhang ${ }^{1}$ Thomas R. Neu ${ }^{2}$ - Yutong Zhang ${ }^{1}$ - Sören Bellenberg ${ }^{1}$. \\ Ute Kuhlicke $^{2} \cdot$ Qian $_{\mathrm{Li}^{1}}{ }^{1}$ Wolfgang Sand ${ }^{1} \cdot$ Mario Vera $^{1}$
}

Published online: 4 August 2015

(C) Springer-Verlag Berlin Heidelberg 2015

\section{Erratum to: Appl Microbiol Biotechnol}

DOI 10.1007/s00253-015-6775-y

The original version of this article contains mistake. The correct presentation of Table 1 is shown below:

Table 1 Characteristics and binding targets of major fluorochromes used in this study

\begin{tabular}{llll}
\hline Fluorochrome & Binding targets & Ex / Em wavelength [nm] & Concentration \\
\hline SYTO 9 & Nucleic acids (NA) & $483 / 500-560$ & $6 \mu \mathrm{M}$ \\
SYTO 64 & NA & $599 / 625-700$ & $6 \mu \mathrm{M}$ \\
SybrGreen & NA & $494 / 510-580$ & $6 \mu \mathrm{M}$ \\
DDAO & Extracellular NA & $630 / 650-700$ & $10 \mu \mathrm{g} / \mathrm{mL}$ \\
DAPI & NA & $405 / 415-490$ & $100 \mu \mathrm{g} / \mathrm{mL}$ \\
SyproRed & Proteins & $550 / 580-650$ & $1 \times$ work solution \\
SyproOrange & Proteins & $475 / 520-610$ & $1 \times$ work solution \\
FM1-43 & Membrane & $480 / 580-650$ & $10 \mu \mathrm{g} / \mathrm{mL}$ \\
FITC-conjugated lectins & EPS glycoconjugates & $490 / 505-600$ & $50-100 \mu \mathrm{g} / \mathrm{mL}$ \\
TRITC- conjugated lectins & EPS glycoconjugates & $555 / 570-650$ & $50-100 \mu \mathrm{g} / \mathrm{mL}$ \\
Calcofluor white M2R & $\beta$-polysaccharides & $405 / 420-460$ & $100 \mu \mathrm{g} / \mathrm{mL}$ \\
Nile red & Lipid-rich domains & $530 / 570-630$ & $10 \mu \mathrm{g} / \mathrm{mL}$ \\
\hline
\end{tabular}

Note: All fluorochromes were from Invitrogen, except Nile red (Sigma Aldrich).

The online version of the original article can be found at http://dx.doi.org/ 10.1007/s00253-015-6775-y.

Mario Vera

mario.vera@uni-due.de

1 Aquatische Biotechnologie, Biofilm Centre, Universität Duisburg - Essen, Universitätsstraße 5, 45141 Essen, Germany

2 Department of River Ecology, Helmholtz Centre for Environmental Research-UFZ, Brückstraße 3a, 39114 Magdeburg, Germany 